\title{
GESTÃo dA CLINICA COMO FERRAMENTA PARA MELHORIAS DOS RESULTADOS ASSISTENCIAIS
}

\section{Clinic management as a tool for improved assistance results}

\author{
Simone Simon ${ }^{1}$, Giseli Cipriano Rodacoski ${ }^{2}$
}

\begin{abstract}
1 Administradora. Graduada em Administração pela UNICESUMAR. Foz do Iguaçu/PR e Especializanda em Gestão Hospitalar - HOSPSUS, Gerente de Qualidade no Hospital Costa Cavalcante.

2 Psicóloga. Mestre em Educação e Doutora em Biotecnologia, Docente na Escola de Saúde Pública do Paraná.

CONTATO: Giseli Cipriano Rodacoski | Email: giseli.rodacoski@sesa.pr.gov.br
\end{abstract}

COMO CITAR Simon S, Rodacoski GC. Gestão da clínica como ferramenta para melhorias dos resultados assistenciais. R. Saúde Públ. Paraná. 2019 Jul;2(Suppl 1): 46-53

CoPYRIGHT Esta obra é disponibilizada nos termos da Licença Creative Commons - 4. 0
Internacional. É permitida a reprodução parcial ou total desta obra, desde que citada a fonte.

RESUMO Centrada na implantação de estratégias de segurança do paciente, a Gestão da Clínica abrange elementos de atenção gerenciada e governança clínica, é indispensável conhecer métodos que contribuam para um gerenciamento excepcional, selecionando bases de pesquisas confiáveis e atualizadas, para que proporcionem efetividade e um diferencial focado no cuidado do paciente dentro das instituições hospitalares. Com isso, se dá a importância do conhecimento e gerenciamento das diretrizes básicas para elaboração e implantação de protocolos, o que é determinante para que todos percebam o seu papel no atingimento dos resultados. Assim, este artigo tem como objetivo o de apresentar um relato de experiência da implantação da Unidade de Gerenciamento de Protocolos em um hospital do interior do Paraná. Os resultados da pesquisa indicam que a referida implantação objetivou institucionalizar e padronizar diretrizes que anteriormente estavam sendo coordenadas por unidades com especificidades diferentes, sendo que atualmente esses documentos são elaborados considerando um modelo 
institucional padrão, o qual inclui uma linguagem clara de todos os envolvidos no processo, e o monitoramento dos resultados é realizado pelos gestores e gerentes através de indicadores com metas mensuráveis e exequíveis. No contexto geral, todos os protocolos do hospital são acompanhados no indicador estratégico "taxa de efetividade geral dos protocolos" que em média é de 95\%. Concluiu-se que a Gestão da Clínica resulta em maior segurança do paciente, considerando que as etapas são ferramentas de melhorias assistenciais, visto que são compostas por capacitação e sensibilização continuada das equipes, otimizando recursos e disseminando uma linguagem íntegra e única.

PALAVRAS-CHAVE: Governança Clínica. Protocolos Clínicos. Assistência Centrada no Paciente.

ABSTRACT Focused on the implementation of patient safety strategies, Clinic Management covers elements of managed care and clinical governance, it is necessary to know methods that contribute to an exceptional management, selecting reliable and updated research bases, to provide effectiveness and a differential focused on the care of patients in hospital institutions. This shows the importance of the knowledge and management of the basic guidelines for elaboration and implementation of protocols, which is determinant to make everyone notice what their role is in the achievement of the results. Thus, this article aims to present an experience report on the implementation of the Protocol Management Unit in a hospital in the countryside of the state of Paraná. The results of the research indicate that this implementation aimed to institutionalize and standardize guidelines that were previously coordinated by units with different specificities. Currently, these documents are elaborated considering a standard institutional model, which includes clear language of all those involved in the process. Results are monitored by managers through indicators with measurable and achievable goals. In the general context, all hospital protocols are tracked according to the strategic indicator "overall protocol effectiveness", which on average is $95 \%$. It was concluded that the Clinic Management results in greater patient safety, considering that the stages are tools of improvement of care, since they consist of continuous training and awareness of the teams, optimizing resources and disseminating a unique and integral language.

KEYWORDS: Clinical Governance. Clinical Protocols. Patient-centered Care.

\section{INTRODUÇÃO}

expressão Gestão da Clínica foi
empregada no Brasil por Mendes ${ }^{1}$ em
2001, trazendo elementos tanto da Atenção Gerenciada, amplamente disseminada nos Estados Unidos nos anos 1980 como Governança Clínica. Segundo o autor, a Gestão da Clínica compreende um conjunto de tecnologias de microgestão aplicáveis ao Sistema Único de Saúde brasileiro que, baseadas nas diretrizes clínicas, utiliza a "gestão da condição de saúde, de gestão de caso, de auditoria clínica e de listas de espera" para prover uma atenção à saúde de qualidade. 
Com o desenvolvimento significativo no modelo de atenção à saúde passando de fragmentado para uma articulação em Redes Assistenciais e dos avanços tecnológicos, surgem as mais variadas opções de protocolos para a condução terapêutica e diagnóstica. ${ }^{1}$

A diversificação dos protocolos muitas vezes não está relacionada às melhores práticas assistenciais, pois tais protocolos podem ter sido elaborados por diretrizes clínicas de má qualidade, baseadas em pesquisas científicas incompletas, mal interpretadas ou desatualizadas, tornando-os ineficazes favorecendo a resistência à adesão por parte de muitos profissionais de saúde, e colocando em risco a segurança do paciente, podendo também ser entendidas como algo restritivo que limita sua autonomia, além de onerar os serviços hospitalares por seguir recomendações e práticas inadequadas.

Com o objetivo de direcionar os esforços no sentido de reduzir riscos e oferecer um cuidado ao paciente com qualidade e segurança, o Ministério da Saúde ${ }^{2}$ define diretrizes, estratégias e critérios de avaliação com base em evidências científicas para orientar a elaboração de protocolos clínicos a serem feitos pelas instituições. O protocolo deve ser elaborado considerando o contexto institucional, e ser apresentado de uma maneira estruturada e com o objetivo de alinhar condutas entre equipes de saúde e usuários.

Os protocolos de forma documental são apresentados por extensos documentos que podem ser classificados em setoriais ou institucionais, sendo desejável que mesmo os setoriais estejam disponíveis para a consulta de todos os atores envolvidos, de modo a favorecer a comunicação e integração do cuidado, podendo ser intitulados como: diretrizes clínicas, protocolos, guidelines, clinical patthways, linhas de cuidados, procedimentos, normas técnicas, etc. São vários os tipos de abordagens, porém para reduzir esta variabilidade de termos, favorecer o entendimento e alinhamento dos conceitos, vamos considerar neste artigo diretrizes clínicas e procedimentos como protocolos respeitando as especificidades, as diferentes realidades de saúde e dos indivíduos sob cuidados ${ }^{3}$.

Muitas são as evidências da literatura quanto aos benefícios da aplicação de protocolos. A literatura aponta vantagens tais como o aumento da efetividade da assistência bem como a segurança do paciente por meio da detecção, prevenção e diminuição da incidência de eventos adversos relacionados ao cuidado.

São importantes instrumentos para alinhar as diferentes formas de prestar assistência, orientados por diretrizes e validados através da pesquisa científica, e envolvem a inclusão de novas tecnologias, medicamentos e ações técnicas, recursos indispensáveis para garantia da qualidade do cuidado?.

O gerenciamento da utilização dos protocolos, de modo que sejam adotados por toda a equipe, demanda habilidade de comunicação e contínua análise quanto à sua efetividade. Quanto mais complexa a situação a ser atendida, mais serviços serão envolvidos e o risco pela falha na comunicação é maior. A identificação de marcadores e indicadores de análise favorece o monitoramento e a rápida ação de melhoria no processo.

Ao considerar a importância da gestão da clínica nos serviços hospitalares, o objetivo deste trabalho foi apresentar experiência exitosa de um hospital no interior do Paraná que alcançou bons resultados assistenciais com a implantação da Unidade de Gerenciamento de Protocolos. 


\section{ANÁLISE CONTEXTUAL}

A gestão da clínica foi implantada em um hospital localizado na cidade sede da $9^{a}$ Regional de Saúde, composta por mais oito municípios da região oeste do Paraná, além de estar situado em região de tríplice fronteira.

Este hospital é de média e alta complexidade, referência para cardiologia - 13\% dos atendimentos - oncologia (14,9\%) e gestação de alto de risco (37,2\%), e realiza em média 600 tipos de cirurgias sendo 400 por mês. Além de ser uma entidade filantrópica, também tem convênio próprio e atende a 49 planos de saúde.

possui 202 leitos, sendo 35 leitos de UTIs, e as contratualizações abrangem demais hospitais da região e clínicas de exames de imagens e laboratoriais. O hospital conquistou em 2014 o certificado de "Acreditado com excelência" na prestação de serviços em todos os setores.

\section{RELATO DE EXPERIÊNCIA}

O Ministério da Saúde instituiu o Programa Nacional de Segurança do Paciente em 2013 devido à observância dos dados provocados às pessoas e dos custos gerados aos serviços de saúde. A segurança do paciente é uma importante discussão no campo das políticas de saúde no mundo e tem sido objeto de diversos programas incentivados pela Organização Mundial de Saúde e mais recentemente pelo Ministério da Saúde no Brasil ${ }^{4}$. Alinhado ao Ministério da Saúde a Instituição cenário deste estudo planejou ações voltadas ao monitoramento dos processos de trabalho com vistas a produzir impacto na segurança do paciente.

A utilização de protocolos setoriais já era uma rotina nas unidades hospitalares, o desafio era monitorar e institucionalizar as ações setoriais, surgindo assim a necessidade de criar a Unidade de Gerenciamento de Protocolos com o objetivo de institucionalizar as diversas diretrizes nas quais estavam sendo coordenados por equipes de acordo com as suas especificidades, mas com a necessidade de fortalecer a comunicação, promover integralidade da assistência e segurança do paciente. Esta unidade assumiu responsabilidade em conduzir o processo de elaboração, implantação e revisão periódicas dos protocolos.

Na etapa de elaboração acontece uma assessoria à equipe técnica para que definam os passos do protocolo com base em uma modelagem institucional. Após a determinação das ações assistenciais pela área técnica da especialidade, os protocolos são avaliados pela Unidade de Gerenciamento composta por uma equipe multiprofissional que verifica se há clareza nas orientações de conduta, se há roteiro lógico no qual está contemplado fluxogramas e/ou algoritmos, marcadores para identificação de não conformidades, indicadores de resultados e efetividade. Fazem parte da equipe multiprofissional o médico, enfermeiro, administrador, farmacêutico nutricionista e fisioterapeuta que fazem a validação dos protocolos quanto à qualidade da comunicação escrita e após esta etapa inicia-se então a fase de implantação.

A fase de implantação consiste em ações de disseminação dos protocolos, incluindo a capacitação das equipes, sensibilização de todos os profissionais envolvidos na sua utilização por meio de reuniões setoriais, comunicações internas, intranet e jornal interno. São realizadas também reuniões de interações entre os processos de 
apoio, para avaliar os gaps que impactam no resultado do protocolo para propor soluções como por exemplo: horário de procedimentos, tempo para realização de exames, tempo de laudo, uso otimizado de recursos e implantação de novas rotinas.

A elaboração, a implantação e a implementação de protocolos clínicos neste hospital são utilizadas como ferramentas para melhoria dos resultados assistenciais, promovendo maior segurança nas decisões clínicas, onde a padronização de condutas resulta na diminuição de eventos adversos, morbidade e mortalidade institucional evitável. É utilizado como estratégia da equipe multiprofissional para o planejamento da assistência centrada no paciente de acordo com as suas necessidades e riscos.

A fase de revisão dos protocolos se dá com a periodicidade de 2 anos, exceto em demandas que se fizerem necessárias novas alterações. O responsável pelo protocolo deverá revisálo juntamente com a equipe multidisciplinar incluindo os membros nos quais o protocolo tenha impacto, caso exista alguma alteração as equipes deverão ser avisadas e treinadas concluindo a disseminação das novas informações.

Desde o início da Unidade de Gerenciamento de Protocolos foram implantados 18 protocolos, sendo que 6 deles fazem parte do Programa Nacional de Segurança do Paciente que são: protocolo de cirurgia segura, prevenção de queda, prevenção de úlcera por pressão, administração segura de medicamento, identificação segura do paciente e higienização das mãos ${ }^{4}$. Nos outros 12 protocolos foram considerados os de maior prevalência, gravidade e/ou risco para serem implantados, que são eles: protocolo de dor torácica, prevenção de infecção de cateter venoso central, protocolo de membrana hialina, prevenção de pneumonia associada à ventilação mecânica, prevenção de sepse adulto e sepse grave neonatal, time de resposta rápida, doença hipertensiva da gestação, prevenção de infecção do trato urinário, tratamento de insuficiência cardíaca, prevenção de hipotermia e protocolo de prevenção de tromboembolismo venoso.

\section{MONITORAMENTO E RESULTADOS}

Realizar o monitoramento tem como propósito subsidiar os gestores com informações mais simples e tempestivas sobre a operação e os efeitos, resumidas em painéis ou sistemas de indicadores de monitoramento, e tem como objetivo auxiliar os gestores no momento adequado para a tomada de decisão com a finalidade de gerar ciclos de melhorias, para isto, faz-se necessário coletar indicadores com periodicidade que permita aos gestores reagir ainda dentro de um período de efetivação. ${ }^{5}$

\footnotetext{
Monitoramento consiste no acompanhamento contínuo, cotidiano, por parte de gestores e gerentes, do desenvolvimento dos programas e políticas em relação a seus objetivos e metas. É uma função inerente à gestão dos programas, devendo ser capaz de prover informações para seus gestores, permitindo a adoção de medidas corretivas para melhorar sua operacionalização. É realizado por meio de indicadores, produzidos regularmente com base em diferentes fontes de dados, que dão aos gestores informações sobre o desempenho de programas, permitindo medir se objetivos e metas estão sendo alcançados ${ }^{6}$.
}

Além disso, é preciso que as informações geradas pelos indicadores sejam apresentadas em formato de rápida visualização e interpretação dos resultados. Bons indicadores precisam ser mensuráveis, alcançáveis, pertinentes, baseados 
em tempo e alinhados a metas estratégicas. O termo indicador é conceituado como:

Uma medida, de ordem quantitativa ou qualitativa, dotada de um significado particular e utilizada para organizar e captar as informações relevantes dos elementos que compõem o objeto da observação. É um recurso metodológico que informa empiricamente sobre a evolução do aspecto observado?.

O hospital faz o monitoramento da efetividade de todos os protocolos por meio do acompanhamento do Indicador Estratégico:
Taxa de Efetividade Geral dos Protocolos que faz parte da Ação Estratégica: Fomentar a Política de Segurança do Paciente, que tem mantido nos últimos dois anos (2016 e 2017) a média de 95\% de Efetividade dos Protocolos, sendo a meta institucional estabelecida de 90\%, e os valores são mensurados em um software de gestão hospitalar:

Este resultado demonstra o esforço e comprometimento diário das equipes multiprofissionais e do hospital pela busca contínua pelas melhores práticas assistenciais baseadas em diretrizes clínicas confiáveis.

Figura 1 Painel - Taxa de Efetividade dos Protocolos - 2016

\begin{tabular}{|l|ll|}
\hline Taxa de Efetividade dos & Previsto & 90,00 \\
Protocolos - Geral & Real & 95,05 \\
\hline
\end{tabular}

\begin{tabular}{|l|ll|}
\hline Taxa de Efetividade do Protocolo de & Previsto & 90,00 \\
Cirurgia Segura & Real & 99,21 \\
\hline Taxa de Efetividade do Protocolo de Dor & Previsto & 90,00 \\
Torácia & Real & 90,90 \\
\hline Taxa de Efetividade do Protocolo de & Previsto & 90,00 \\
ICVC & Real & 94,64 \\
\hline Taxa de Efetividade do Protocolo de & Previsto & 90,00 \\
Membrana Hialina & Real & 98,79 \\
\hline Taxa de Efetividade do Protocolo de PAV & Previsto & 90,00 \\
\hline Taxa de Efetividade do Protocolo de & Previsto & 90,00 \\
Prevenção de Queda & Real & 99,91 \\
\hline Taxa de Efetividade do Protocolo de & Previsto & 90,00 \\
Prevenção de Sepse & Real & 89,27 \\
\hline Taxa de Efetividade do Protocolo de & Previsto & 90,00 \\
\hline Prevenção de Úlcera por Pressão & Real & 99,48 \\
\hline Taxa de Efetividade do Protocolo de & Previsto & 90,00 \\
\hline Prevenção de Sepse Grave N. & Real & 95,86 \\
\hline Time de Resposta Rápida & Previsto & 90,00 \\
\hline Doenças Hipertensivas na Gestação & Real \\
\hline
\end{tabular}

Fonte: Dados da pesquisa 
Figura 2: Painel - Taxa de Efetividade dos Protocolos - 2017

\begin{tabular}{|c|c|c|c|c|}
\hline & & $\begin{array}{l}\text { Taxa de Efetividade do Protocolo de } \\
\text { Cirurgia Segura }\end{array}$ & $\begin{array}{l}\text { Previsto } \\
\text { Real }\end{array}$ & $\begin{array}{l}90,00 \\
99,16\end{array}$ \\
\hline & & $\begin{array}{l}\text { Taxa de Efetividade do Protocolo de Dor } \\
\text { Torácia }\end{array}$ & $\begin{array}{l}\text { Previsto } \\
\text { Real }\end{array}$ & $\begin{array}{l}90,00 \\
93,38\end{array}$ \\
\hline & & $\left\{\begin{array}{l}\text { Taxa de Efetividade do Protocolo de } \\
\text { ICVC }\end{array}\right.$ & $\begin{array}{l}\text { Previsto } \\
\text { Real }\end{array}$ & $\begin{array}{l}90,00 \\
91,78\end{array}$ \\
\hline & & $\left\{\begin{array}{l}\text { Taxa de Efetividade do Protocolo de } \\
\text { Membrana Hialina }\end{array}\right.$ & $\begin{array}{l}\text { Previsto } \\
\text { Real }\end{array}$ & $\begin{array}{l}90,00 \\
99,01\end{array}$ \\
\hline & & Taxa de Efetividade do Protocolo de PAV & $\begin{array}{l}\text { Previsto } \\
\text { Real }\end{array}$ & $\begin{array}{l}90,00 \\
95,40\end{array}$ \\
\hline $\begin{array}{l}\text { Taxa de Efetividade dos } \\
\text { Protocolos - Geral }\end{array}$ & $\begin{array}{ll}\text { Previsto } & 90,00 \\
\text { Real } & 95,75\end{array}$ & $\left\{\begin{array}{l}\text { Taxa de Efetividade do Protocolo de } \\
\text { Prevenção de Queda }\end{array}\right.$ & $\begin{array}{l}\text { Previsto } \\
\text { Real }\end{array}$ & $\begin{array}{l}90,00 \\
99,88\end{array}$ \\
\hline & & $\begin{array}{l}\text { Taxa de Efetividade do Protocolo de } \\
\text { Prevenção de Sepse }\end{array}$ & $\begin{array}{l}\text { Previsto } \\
\text { Real }\end{array}$ & $\begin{array}{l}90,00 \\
94,09\end{array}$ \\
\hline & & $\left\{\begin{array}{l}\text { Taxa de Efetividade do Protocolo de } \\
\text { Prevenção de Úlcera por Pressão }\end{array}\right.$ & $\begin{array}{l}\text { Previsto } \\
\text { Real }\end{array}$ & $\begin{array}{l}90,00 \\
99,60\end{array}$ \\
\hline & & $\left\{\begin{array}{l}\text { Taxa de Efetividade do Protocolo de } \\
\text { Prevenção de Sepse Grave N. }\end{array}\right.$ & $\begin{array}{l}\text { Previsto } \\
\text { Real }\end{array}$ & $\begin{array}{l}90,00 \\
96,34\end{array}$ \\
\hline & & $\left\{\begin{array}{l}\text { Taxa de Efetividade do Protocolo do } \\
\text { Time de Resposta Rápida }\end{array}\right.$ & $\begin{array}{l}\text { Previsto } \\
\text { Real }\end{array}$ & $\begin{array}{l}90,00 \\
95,15\end{array}$ \\
\hline & & $\begin{array}{l}\text { Taxa de Efetividade do Protocolo de } \\
\text { Doenças Hipertensivas na Gestação }\end{array}$ & $\begin{array}{l}\text { Previsto } \\
\text { Real }\end{array}$ & $\begin{array}{l}90,00 \\
92,20\end{array}$ \\
\hline & & $\begin{array}{l}\text { Taxa de Efetividade do Protocolo de } \\
\text { Prevenção de Infecção do Trato Urinário }\end{array}$ & $\begin{array}{l}\text { Previsto } \\
\text { Real }\end{array}$ & $\begin{array}{l}90,00 \\
93,07\end{array}$ \\
\hline
\end{tabular}

Fonte: Dados da pesquisa.

\section{CONSIDERAÇÕES FINAIS}

Do ponto de vista organizacional, a implantação e a implementação de protocolos neste hospital proporcionaram maior integralidade entre as equipes, comunicação efetiva, padronização das condutas clínicas, redução das variações do cuidado, os recursos passaram a ser utilizados de maneira adequada e otimizada, tornaram mais evidentes as barreiras de segurança refletindo em um melhor desfecho clínico, as não-conformidades passaram a ser detectadas precocemente por meio de gatilhos e/ou erros e auxiliaram na disseminação da cultura de segurança institucional.

No entanto é frequente haver problemas 
para sua implantação ou implementação nas instituições hospitalares, e, para que estratégias de segurança do paciente sejam efetivas, o conhecimento pelos profissionais, treinamentos e a adoção dos protocolos e diretrizes clínicas são condições básicas quanto à disponibilidade de materiais e equipamentos para o seu cumprimento ${ }^{8}$.

O processo melhorou a compreensão do hospital referente à atuação da educação permanente, e passou a considerá-la a principal ferramenta para as mudanças nos processos de trabalho. Nesse sentido, intervenções educacionais contínuas, concentradas nos protocolos com ações respaldadas em evidências, ajudam a mudar padrões e garantir a adesão e a efetividade dos protocolos ${ }^{9}$

\section{REFERÊNCIAS}

1. Mendes EV. As Redes de Atenção à Saúde. 2. ed. Brasília: Organização Pan- Americana da Saúde: 2011.

2. Ministério da Saúde (BR). Glossário Temático: Economia da Saúde. Brasília: Ministério da Saúde; 2005

3. Vecina Neto G. Malik AM. Gestão em Saúde. 2. ed. Rio de Janeiro: Guanabara Koogan; 2011.

4. Ministério da Saúde (BR). Portaria n 529 de 1 de abril de 2013 Institui o Programa Nacional de Segurança do Paciente. Brasília: Ministério da Saúde: 2013

5. Jannuzzi PM. Avaliação de programas sociais no Brasil: repensando práticas e metodologias das pesquisas avaliativas. Planejamento e Políticas públicas. Brasília, DF, v. 36, p. 251-275, 2011.

6. Vaitsman J, Rodrigues RWS, Paes-Sousa R. O Sistema de avaliação e monitoramento das políticas e programas sociais: a experiência do Ministério do Desenvolvimento Social e combate à fome do Brasil. UNESCO: 2006.

7. Ferreira H, Cassiolato M, Gonzalez R. Uma experiência de desenvolvimento metodológico para avaliação de programas: o modelo lógico do programa segundo tempo. [Texto para discussão]. Rio de Janeiro: IPEA, 2009

8. Vendramini RCR, da Silva EA, Ferreira KASL, Possari JF, Baia WRM. Segurança do paciente em cirurgia oncológica: experiência do Instituto do Câncer do Estado de São Paulo. Rev. Esc. Enferm. USP. 2010 [acesso em 22/10/2018]: volume (44) 3:75. Disponível em:<http://www.scielo.br/scielo.php?script=sci_ arttext\&pid=S0080- 62342010000300039>

9. Oliveira RM, Leitão IMTA, Silva LMS, Figueiredo SV, Sampaio RL, Gondim MM. Estratégias para promover segurança do paciente: da identificação dos riscos às práticas baseadas em evidências. Esc. Anna Nery. 2014; 18(1): 122-129. 